\title{
Herramientas de mercadeo tradicional y digital utilizadas por grandes empresas ubicadas en países en vías de desarrollo y su relación con el desempeño exportador: el caso de Costa Rica una perspectiva desde la gerencia en las mipymes
}

Cómo citar este artículo / To reference this article / Comment citer cet article / Para citar este artigo:

Quirós-Gómez,J.; Arce-Gutiérrez, S. (2020). Herramientas de mercadeo tradicional y digital utilizadas por grandes empresas ubicadas en países en vías de desarrollo y su relación con el desempeño exportador: el caso de Costa Rica. Revista Escuela de Administración de Negocios, (89), 33-50. DOI: https://doi.org/10.21158/01208160.n89.2020.2817

\section{Resumen}

Las exportaciones juegan un papel clave en el desarrollo económico de los países, estas se han visto beneficiadas por el uso de internet, según estudios previos. No obstante, la literatura no es clara con respecto a la relación que existe entre las herramientas de mercadeo digital y tradicional con el desempeño exportador de grandes empresas ubicadas en países en vías de desarrollo. Con el fin de determinar si existe esta relación y, además, identificar cuáles herramientas poseen un mayor impacto en este tipo de organizaciones, se desarrolla la presente investigación. Se basa en una metodología cualitativa por medio de entrevistas a profundidad realizadas a empresas con más de cien colaboradores ubicadas en Costa Rica, las cuales fueron analizadas con el software Atlas.TI8. Los resultados apuntan a que efectivamente existe la posible relación descrita, y cómo, a pesar de que se evidencia la relevancia que tienen las herramientas de mercadeo digital en la actualidad, existe una predominancia de las herramientas tradicionales. El análisis permite concluir que cada vez son más las compañías que manejan las herramientas de mercadeo digital a nivel interno dentro la organización, debido a la importancia de estas para el logro de los objetivos, además fue posible identificar que las empresas estudiadas han logrado un equilibrio entre mantener las actividades de mercadeo tradicional e incursionar en el uso del mercado digital.

Palabras clave: mercadeo; mercadeo digital; herramientas de mercadeo digital; mercadeo tradicional; exportaciones; desempeño exportador; Costa Rica.

\footnotetext{
Bachiller en Administración de Empresas y Especialista en Administración de Proyectos. Escuela de Administración de Empresas, Tecnológico de Costa Rica. ORCID: https://orcid.org/0000-0003-4569-9532

2 Licenciada en Administración de Empresas con énfasis en Finanzas, docente e investigadora. Escuela de Administración de Empresas, Tecnológico de Costa Rica. ORCID: https://orcid.org/0000-0001-8978-9623
} 


\title{
Traditional and digital marketing tools used by large companies in developing countries and their relationship with export performance: the case of Costa Rica
}

\begin{abstract}
Exports play a key role in the economic development of countries, and according to previous studies, they have benefited from the use of Internet. However, the literature is not clear regarding the relationship between digital and traditional marketing tools and the export performance of large companies in developing countries. The present research is developed in order to determine whether this relationship exists, and to identify which tools have a greater impact on this type of organizations. This study is based on qualitative information from in-depth interviews carried out in companies with over one hundred collaborators in Costa Rica, and analyzed with the software ATLAS.ti 8. The results show that indeed the relationship described exists, and that, despite the relevance of the digital marketing tools today, what predominates is the traditional tools. The analysis allows to conclude that there is a growing number of companies managing digital marketing tools within the organization, as they are especially important for achieving their goals; it was also possible to identify that the companies under study have reached a balance between maintaining traditional marketing activities and venturing into the use of digital marketing.
\end{abstract}

Keywords: marketing; digital marketing; digital marketing tools; traditional marketing; exports; export performance; Costa Rica.

\section{Ferramentas de marketing tradicional e digital utilizadas por grandes empresas localizadas em países em desenvolvimento e sua relação como desempenho exportador: o caso da Costa Rica}

\section{Resumo}

As exportações desempenham um papel fundamental no desenvolvimento econômico dos países. Elas se beneficiaram com o uso da internet, de acordo com estudos anteriores. No entanto, a literatura não é clara a respeito da relação entre as ferramentas de marketing digital e tradicional com o desempenho exportador de grandes empresas localizadas em países em desenvolvimento. Esta pesquisa foi realizada com o objetivo de determinar se existe essa relação e, além disso, identificar quais ferramentas têm um maior impacto neste tipo de organização. Baseia-se em uma metodologia qualitativa por meio de entrevistas em profundidade realizadas com empresas com mais de cem colaboradores localizadas na Costa Rica, as quais foram analisadas com o software Atlas.TI8. Os resultados mostram que realmente existe a possível relação descrita, e como, apesar da relevância das ferramentas de marketing digital ser evidente hoje, há um predomínio das ferramentas tradicionais. A análise permite concluir que existem cada vez mais empresas que lidam com ferramentas de marketing digital internamente dentro da organização, devido à importância destas para o cumprimento dos objetivos, também foi possível identificar que as empresas estudadas têm alcançado um equilíbrio entre manter as atividades do marketing tradicional e lançar-se no uso do mercado digital.

Palavras-chave: marketing; marketing digital; ferramentas de marketing digital; marketing tradicional; exportações; desempenho de exportação; Costa Rica. 


\section{Relations entre outils du marketing traditionnel et numérique utilisés par les grandes entreprises des pays en développement et performances à l'exportation : le cas du Costa Rica}

\section{Résumé}

Selon plusieurs études, les entreprises exportatrices, qui jouent un rôle clé dans le développement économique des pays, ont su tirer profit de l'utilisation mercatique d'Internet. Ces études manquent cependant de clarté sur la relation entre outils marketing numériques et traditionnels et performances à l'exportation des grandes entreprises des pays en développement. Cette recherche tentera de déterminer si une telle relation existe afin d'identifier quels sont les outils ayant le plus d'impact sur ce type d'entreprises. Cette étude repose sur une méthodologie qualitative réalisée au travers d'entretiens approfondis, analysés avec le logiciel Atlas.TI8, et menés auprès d'entreprises de plus de cent collaborateurs situés au Costa Rica. Les résultats suggèrent que la relation décrite antérieurement existe bel et bien, mais que, même si la pertinence des outils marketing et numérique est évidente aujourd'hui, il existe une prédominance des outils traditionnels. L'analyse permet de conclure que de plus en plus d'entreprises gèrent les outils de marketing numérique en interne, au sein de l'organisation, en raison de leur importance pour la réalisation des objectifs et que, les entreprises étudiées ont su trouver un équilibre entre le maintien d'activités de marketing traditionnel et utilisation du marché numérique.

Mots-clés: marketing; marketing numérique; outils de marketing numérique; marketing traditionnel; exportations; performance à l'exportation; Costa Rica. 


\section{Introducción}

$\mathrm{T}$ al como lo afirman Khan et al. (2012), las exportaciones juegan un rol clave en la prosperidad de la economía, concepto que se refiere a los bienes y los servicios que se producen en el mercado doméstico y se venden a compradores ubicados en otros países. Según la Promotora de Comercio Exterior de Costa Rica (2019), para el 2016 el 29 \% de las compañías exportadoras eran grandes empresas, con una participación del $84 \%$ en el valor total de las exportaciones; sin embargo, ambos porcentajes disminuyeron en comparación con el 2017.

En este mismo orden de ideas, Tan y Sousa (2015) explican que, debido a los avances de la globalización y la competencia internacional, un gran número de empresas ha decidido iniciar sus actividades de exportación. En este sentido, Van Dijk (2002) comenta que la exportación constituye una de las principales herramientas para que los países en vía de desarrollo puedan establecer una relación con la economía mundial y reciban así beneficios de países desarrollados.

Tan y Sousa (2015) también mencionan que el mercadeo es uno de los aspectos fundamentales en el fomento de las exportaciones, dado que permite entender las preferencias del consumidor y superar los esfuerzos que realiza la competencia en mercados internacionales. Sumado a lo anterior, Ledermana, Olarreaga y Zavala (2016) explican que la promoción de las exportaciones ayuda a las empresas a introducirse y sobrevivir en los mercados en los que estas desean incursionar, lo cual forma parte de las actividades realizadas desde el área de mercadeo.

Por su parte, Yogesh, Sharaha y Roopan (2019) mencionan que el desarrollo de las tecnologías de la información y los avances en las herramientas de comunicación digital impulsan a las empresas a modificar la forma en la que venden sus productos. En este sentido, Diez, Blanco y Prado (2019) explican que el mercadeo digital ha transformado la forma en la que las organizaciones mantienen la comunicación con sus clientes alrededor del mundo, de manera que esto es un factor clave para enfrentar los retos derivados del mercadeo.

Anato (2006) menciona que el «internet y otros medios digitales facilitan la comunicación interactiva que no es posible a través del uso del marketing convencional» (p. 12).Asímismo, Bianchiy Mathews (2016) relacionan lo anterior con las exportaciones, puesto que los autores afirman que internet facilita el desarrollo de redes comerciales en mercados internacionales, así como el desarrollo de otro tipo de actividades que realizan las empresas con respecto a este tema.

Yamin (2017) menciona que el mercadeo digital lo utilizan las compañías para apoyar actividades que busquen conocer a sus clientes a través de buscadores, redes sociales, correo electrónico y páginas web, entre otras herramientas; de forma que se puedan satisfacer las necesidades y los deseos de sus clientes actuales y potenciales, quienes utilizan dispositivos digitales en sus actividades cotidianas. Así mismo, el autor explica que algunos beneficios de estas herramientas son la personalización, la velocidad y la accesibilidad de la información. Según Bala y Gomathi (2017), el mercadeo digital juega un papel fundamental en el mundo competitivo de la actualidad, de modo que lo utilizan todo tipo de organizaciones. Por su parte, Anato (2006) expresa que las compañías deben esforzarse por utilizar los medios digitales para desarrollar una comunicación de tipo personal $y$, de esta forma, establecer relaciones a largo plazo con los clientes mediante la interacción a través de comunidades virtuales.

No obstante, Yamin (2017) recalca que, a fin de llevar a cabo los negocios de forma exitosa, se requiere de métodos convencionales y modernos, y son los segundos los que tienen una mayor influencia. Raluca (2016) propone que las compañías no deben ignorar las herramientas de mercadeo tradicionales, sino 
que las deben combinar con mercadeo digital, con el propósito de alcanzar los objetivos de la organización; la autora explica que el mercadeo tradicional incluye todas aquellas herramientas que no utilicen medios digitales, tales como anuncios en la radio, vallas publicitarias, folletos, llamadas telefónicas y anuncios de televisión, entre otros.

Si se toma como base lo anterior, el problema que busca solventar el presente artículo se delimita en determinar si existe una posible relación entre el desempeño exportador de grandes empresas ubicadas en países en vía de desarrollo, tal como Costa Rica, y las herramientas de mercadeo tradicional y digital que estas utilizan, dado que, según la literatura consultada, no existe un estudio similar para el país que logre guiar a las empresas sobre cómo dirigir sus esfuerzos en mercadeo en el propósito de impulsar su internacionalización y así lograr dar solución a la irregularidad del nivel de exportaciones de las grandes empresas entre un año y otro, mediante el uso de herramientas digitales y tradicionales de mercadeo.

\section{Revisión de literatura}

\subsection{Mercadeo}

$\mathrm{D}$ e acuerdo con Gervase (2009), el concepto de mercadeo se refiere al proceso de concepción, fijación de precios, promoción y distribución de productos, con el fin de llevar a cabo intercambios que satisfagan los objetivos del individuo y de la organización. De igual forma, Moreno (2007) manifiesta que el mercadeo «es un esfuerzo de intercambio centrado en el mercado, orientado al consumidor y dirigido a través de su coordinación a generar satisfacción en los clientes como llave para cumplir los objetivos de las organizaciones» (p. 1).

Aunado a lo anterior, Gervase (2009) considera que el mercadeo busca anticipar y satisfacer las necesidades de los clientes a través de procesos de intercambio beneficiosos para estos y las organizaciones, de una forma más rentable y eficaz que la competencia; por su parte, Yamin (2017) sostiene que el objetivo del mercadeo es proporcionar información sobre las necesidades del mercado, la experiencia del cliente, los movimientos de la competencia y las tendencias del mercado. En el mismo orden de ideas, es importante definir qué es una estrategia de mercadeo, la cual, de acuerdo con Castellano y Urdaneta (2015), «incluye de manera secuencial la medición de la demanda, su segmentación y orientación, y finalmente la tarea de posicionar el producto o servicio, que puede traer ventajas competitivas para la organización» (p. 481).
Ahora bien, Mohasoa (2014) refuerza lo anterior al mencionar que el mercadeo tradicional pretende persuadir a los clientes mostrándoles las características y beneficios de un bien o servicio a través de medios tradicionales; García-Marín (2015) explica que algunos de estos medios son «televisión, radio, publicidad al aire libre, folletos, puntos impresos publicitarios, correo directo y recompensa en tiendas» (p. 48). Por su parte, Raluca (2016) destaca que un beneficio de este tipo de mercadeo es la durabilidad, puesto que la información suele mantenerse por más tiempo. Sin embargo, la autora recalca que, a fin de obtener mejores resultados, se debe asegurar que los medios estén en el lugar adecuado y dirigidos al público correcto.

Bianchi y Mathews (2016) mencionan que el uso de internet en las actividades que se realizan en una empresa genera una mejora notable en su desempeño. Por este motivo, Moschini (2012) coincide en que hoy las empresas apuestan por una mayor cantidad de recursos a sus estrategias de mercadeo en medios digitales, de forma que estas se adapten a las necesidades actuales de los consumidores, aumenten su visibilidad, mejoren su imagen pública y optimicen la relación con los clientes internos y externos. 
Por su parte, Yogesh, Sharaha y Roopan (2019) definen el mercadeo digital como aquel que se realiza mediante el uso de tecnologías digitales que se encuentran, principalmente, en internet. A su vez, Gómez (2013) afirma que el principal objetivo de este tipo de mercadeo es que las empresas se comuniquen con clientes actuales $\mathrm{y} / \mathrm{o}$ potenciales con un motivo comercial, mediante el uso de tecnologías de información y dispositivos con acceso a estas. El autor destaca que cada día son más las personas que tienen acceso a internet, por lo cual las organizaciones deben tomar ventaja de esto para «mejorar su imagen corporativa, comunicarse con sus clientes, mantener informados a los consumidores, ofrecer diversos productos y servicios, ampliar su participación en el mercado, $y$, por lo tanto, incrementar sus ventas» ( $\mathrm{p}$. $35)$.

De acuerdo con lo anterior, García-Marín (2015) sostiene que el mercadeo digital se utiliza en la organización para «captar, comunicar, interactuar, participar, probar e incluso vender y obtener realimentación y patrocinadores de productos y servicios» (p. 48). Un ejemplo de esto es la pauta online, la cual Vanegas (2012) plantea que busca dos objetivos principales: generar una mayor recordación de la marca por parte de los usuarios, así como promover $y$ aumentar las ventas.

Por su parte, Maqueira y Bruque (2012) exponen que el uso de herramientas digitales en internet ha venido a darle un giro a la forma en la que los seres humanos se relacionan entre sí y, por ende, también ha modificado la forma en la que las empresas y sus clientes se comunican. Moschini (2012) contextualiza que años atrás era la organización quien decidía cómo, cuándo y por qué se comunicaba con sus clientes, sin embargo, actualmente son estos últimos quienes tienen el poder de tomar las decisiones.

Además, Vanegas (2012) indica que el uso de internet en las actividades de la organización tiene una serie de beneficios, los cuales ponen en ventaja el mercadeo digital con respecto al tradicional. Sin embargo, Gregory, Viet-Ngo y Karavdic (2019) explican que el proceso de transformación de los mercados tradicionales hacia los electrónicos requiere que las compañías tengan capacidades de mercadeo especializadas. En este sentido, el autor explica que el mercadeo digital es interactivo, transaccional, personalizable, medible, barato y flexible.

En este sentido, Yamin (2017) explica que existen diversas herramientas de mercadeo digital, como las redes sociales, por ejemplo, las cuales han transformado las actitudes y las percepciones del mercado objetivo de la organización. El autor expone que estas herramientas han impactado de forma directa la relación entre una marca y el consumidor. Así mismo, Grávalos (2013) expresa que la página web constituye otra herramienta de mercadeo digital que se puede utilizar, la cual tiene dos fines específicos: e-business y e-commerce. El primero busca mejorar los procesos de negocio y, por ende, obtener mejores resultados, mientras que segundo involucra la venta de bienes y servicios. Finalmente, Vanegas (2012) comenta que el email-marketing es otra de las herramientas, cuyo fin es «realizar acciones coordinadas de envío de mensajes personalizados a una base de datos para realizar acciones de marketing directo» (pp. 30-31).

En el mismo orden de ideas, García-Marín (2015) expresa que el mercadeo digital se puede analizar desde tres puntos de vista. Se inicia por el enfoque en el consumidor, en el cual se analizan sus características y el uso que este le brinda a los medios digitales. El segundo punto de vista se centra en la agencia de mercadeo digital, la cual conoce las distintas herramientas que se pueden utilizar para aprovechar al máximo la inversión realizada. Finalmente, el punto restante hace énfasis en la empresa propietaria del bien o servicio, la cual espera obtener un retorno de la inversión realizada en estos medios.

No obstante, Bala y Gomathi (2017) consideran que existen algunos retos que debe enfrentar el mercadeo digital, por ejemplo, el hecho de no perderse dentro de la competencia, debido a que cada día son más las empresas que lo utilizan. Según los autores, otro de los retos es el hecho de compartir contenido que le interese a la audiencia, la cual debe ser 
correctamente seleccionada; además, es necesario que las organizaciones se mantengan actualizadas con respecto a las tendencias que surgen, las cuales varían de forma acelerada. Por su parte, Rodríguez, Camargo, González y Gorrin (2015) explican que las organizaciones deben cumplir con las exigencias de la sociedad actual, la cual se encuentra en búsqueda de respuestas en tiempo real.

Así mismo, Rodríguez et al. (2015) coinciden en que uno de los beneficios de utilizar herramientas de mercadeo digital radica en la medición de los resultados, puesto que estas brindan estadísticas completas sobre las interacciones con los clientes. En este sentido, Vanegas (2012) explica que con la implementación de medios digitales en las actividades de la empresa se puede conocer el retorno de la inversión y generar múltiples indicadores y métricas en tiempo real, lo cual permite tomar decisiones orientadas a la maximización de los resultados; en contraparte, el autor explica que también existen sistemas para medir los resultados de los medios convencionales, sin embargo, estos poseen una menor precisión y requieren de un mayor esfuerzo.

Por su parte, Yogesh, Sharaha y Roopan (2019) sostienen que el mercadeo digital superará las estrategias de mercadeo convencionales, debido a que actualmente las personas prefieren el contenido online. Sin embargo, García-Marín (2015) difiere con lo anterior y menciona que «no todas las personas están familiarizadas con estas tecnologías. Por lo tanto, muchas empresas utilizan una combinación de canales de comercialización tradicionales y digitales para ampliar su comunicación» (p. 48). La idea anterior la apoya Raluca (2016), quien recomienda a las organizaciones estrategias que combinen medios tradicionales y digitales, puesto que, a pesar de que el uso de medios digitales ha aumentado en los últimos años, una parte significativa de los clientes prefiere los medios convencionales.

\subsection{Mercadeo digital, redes de negocio y exportaciones}

Según Cortés (2011), «el marketing a nivel mundial ha evolucionado rápidamente en las últimas décadas, diversificando sus enfoques, técnicas y herramientas» (p. 6). Por su parte, Jiménez (2010) afirma que el mundo actual es un mercado globalizado, por lo cual las empresas deben enfocar sus esfuerzos de mercadeo hacia un mercado internacional; para esto, Peláez y Rodenes (2009) mencionan que las alianzas estratégicas juegan un papel fundamental, puesto que las organizaciones forman parte de un sistema de redes con otras entidades que son clave para su desempeño a nivel internacional, principalmente, por «el acceso a nuevos mercados, la búsqueda de oportunidades y la eficiencia» (p. 113).

Agregado a lo anterior, Cobo y González (2007) mencionan que las alianzas estratégicas son también clave para las organizaciones, debido a que estas «provocan un cambio en las condiciones de la competencia, haciendo que las empresas deban gestionar las relaciones con las organizaciones de su entorno de una manera consciente, profesional y planificada» (p. 558). Apoyando lo anterior, Bianchi y Mathews (2016) explican que el uso de internet beneficia el desarrollo de redes de negocio en mercados internacionales.

Al reforzar lo anterior Puente-Riofrío y AndradeDomínguez (2016) destacan que, debido a la competitividad de los productos en el mercado actual, «se hace necesario que las empresas realicen esfuerzos organizacionales para obtener una mayor participación en el mercado, a través de la diversificación de la cartera de productos con miras al incremento de su rentabilidad» (p. 74). ArgüellesVélez y Benavides-González (2008) explican que el acceso a recursos tecnológicos permite crear una ventaja competitiva con respecto a la competencia, puesto que estos cada vez son más necesarios e indispensables en los procesos de producción; con respecto a este tema, Yamin (2017) destaca cómo, con el uso de herramientas de mercadeo digital, se pueden conocer los esfuerzos que realiza la competencia, lo cual es de utilidad en el momento de dirigir las actividades de mercadeo y tomar decisiones.

Por otra parte, Granada (2012) explica que mantener una relación a largo plazo con los consumidores permite que estos prefieran ciertas empresas por 
encima de la competencia, lo cual se logra a través de una buena comunicación y del cumplimiento de las responsabilidades adquiridas. En este mismo sentido, Agüero (2014) explica que captar nuevos clientes implica que las empresas realicen esfuerzos para que estos deseen formar parte de ellas, a través de acercamiento y el convencimiento.

De acuerdo con Gregory, Viet-Ngo y Karavdic (2019), como parte de estos esfuerzos el uso de tecnologías de la información permite mejorar la comunicación con los consumidores, además de mejorar los procesos de compra y distribución de productos, así como la cadena de suministro. Según los autores, algunas empresas exportadoras aprovechan estos medios para administrar los sistemas de la cadena de suministros, de manera que amplían la coordinación con los canales de distribución y las alianzas con otras organizaciones. Con relación a lo anterior, Vásquez (2009) señala que los canales de distribución buscan «satisfacer los deseos ligados al consumo en las mejores condiciones de lugar, tiempo, calidad, precio y presentación, colocados al menor costo posible y de la forma más eficiente» (p. 10). De igual forma, Asare, Brashear y Jun (2013) mencionan que las actividades que se llevan a cabo para la búsqueda, selección y gestión de los proveedores pueden generarle una serie de beneficios a las organizaciones.

Por consiguiente, Kotler (2002) menciona que comúnmente las empresas desean aumentar su nivel de ventas y, por ende, su utilidad, a través de la búsqueda de nuevos negocios. Para esto, el autor señala que las compañías tienen tres opciones: identificar oportunidades que permitan un crecimiento adicional con los negocios actuales; buscar oportunidades relacionadas con el negocio actual; y finalmente, incursionar en el desarrollo de negocios no relacionados con los actuales.

Con relación a esto Galindo y Viridiana (2015) indican que «la importancia de las exportaciones reside en el efecto que tienen sobre el crecimiento económico, la productividad y el empleo del país en cuestión» (p. 2). No obstante, Hurtado y Castillo (2013) mencionan que «existen diversos factores actitudinales, estructurales y operacionales que impiden que muchas empresas inicien, desarrollen o mantengan las actividades exportadoras» (p. 40). Así mismo, Galindo y Viridiana (2015) comentan que el hecho de considerar el mundo como un único mercado, es decir, ampliar más allá de las fronteras el segmento al que la empresa dirige sus operaciones, es un reto que las compañías deben superar con una estrategia adecuada, la cual debe considerar aspectos culturales, políticos, económicos y sociales.

La idea anterior la apoyan García y Avella (2007), quienes explican que existen algunas trabas que impiden el crecimiento de las exportaciones, por ejemplo, las características del mercado doméstico de la empresa, la falta de información y de conocimiento, el riesgo que implica tomar las decisiones y, principalmente, la falta de recursos. Por su parte, Argüelles-Vélez y Benavides-González (2008) mencionan que el crecimiento económico de los países, en la actualidad, se relaciona en menor medida con los recursos de tipo tradicional, de modo que se le da una mayor prioridad a la tecnología y a la formación de redes, entre otros. Según las autoras, por este motivo los países en vía de desarrollo deben apostar a la adopción de esfuerzos que faciliten el acceso a este tipo de recursos.

Gregory et al. (2019) refuerzan lo anterior comentando que, debido a los avances en la comunicación, los medios de transporte y las tecnologías de la información, se espera que el desempeño de las exportaciones se incremente de manera significativa con el paso del tiempo. En este sentido, los autores explican que el desempeño exportador se refiere al grado en el que los objetivos de la organización se logran con relación a la exportación de un producto o servicio en un mercado internacional, mediante la estrategia de mercadeo de exportación, además de la planificación y la ejecución.

Por su parte, Bianchi y Mathews (2016) mencionan que el uso de internet apoya a las organizaciones para expandir sus actividades de exportación y así dirigirlas de una forma más eficiente, además de reforzar los esfuerzos de mercadeo para exportación que estas 
llevan a cabo mediante el fortalecimiento de la publicidad, las ventas y las investigaciones de mercado. Lo anterior mejora la disponibilidad de la información en mercados internacionales, incrementando la velocidad con la que se internacionaliza la empresa y reduciendo los costos asociados a este proceso.

Finalmente, Gregory et al. (2019) explican que actualmente existe una falta de conocimiento con respecto a la forma en la que se pueden integrar recursos y tecnologías de la información con miras a mejorar la eficiencia del mercadeo y el rendimiento de las exportaciones. Por otra parte, los autores explican que las actividades de mercadeo en la exportación se ven beneficiadas con el uso de recursos electrónicos. Además, Tan y Sousa (2015) concluyen que las compañías exportadoras requieren desarrollar conocimiento y habilidades para diseñar y crear nuevos productos, responder a los cambios del mercado y manejar las relaciones con los clientes; tareas que llevan a cabo en el área de mercadeo.

\section{Metodología}

A fin de cumplir con el objetivo de analizar Las herramientas de mercadeo tradicional y digital utilizadas por grandes empresas ubicadas en países en vías de desarrollo y su relación con el desempeño exportador, en específico para el caso de Costa Rica, este estudio se desarrolla por medio de un enfoque cualitativo, debido a que, tal como lo explican Hernández, Fernández y Baptista (2014), en este tipo de investigación se inicia por examinar los hechos en sí, y en el proceso se desarrolla una teoría coherente con el fin de representar lo que se observa, es decir, se procede a analizar caso por caso hasta llegar a una perspectiva general, de modo que se realiza una exploración desde el punto de vista de los participantes.

En cuanto al alcance de la investigación, se puede afirmar que es descriptivo, puesto que, según mencionan los autores, se busca identificar las características del fenómeno o la situación que se estudie, en la cual se pretende describir la relación que existe entre el desempeño exportador de grandes empresas ubicadas en países en vía de desarrollo y las herramientas de mercadeo tradicional y digital que estas utilizan, específicamente para el caso de Costa Rica.

La recolección de la información se realizó a través de entrevistas a profundidad. La guía de entrevista utilizada se divide en cuatro secciones: información de la empresa — cuatro ítems-, actividades de mercadeo — cuatro ítems —, actividades de mercadeo digital — siete ítems - y desempeño exportador —ocho ítems-; esto si se consideran preguntas de opinión, conocimientos técnicos y antecedentes. Este instrumento se aplicó a una persona de cada compañía participante, funcionaria del departamento de mercadeo o comercial. Para la elaboración de esta guía se tomó como base el artículo publicado por Bianchi y Mathews (2016), en el cual se analiza la interacción de las capacidades del mercadeo en internet con las redes de negocio, la información de exportación disponible y el desempeño exportador.

La base de datos utilizada la brindó la Promotora de Comercio Exterior de Costa Rica, en la que se detalla la información de 2610 compañías exportadoras con más de cien colaboradores, de las cuales, mediante un muestreo por conveniencia, se seleccionaron dieciséis empresas basándose en el principio de Berteaux (1981) para las investigaciones cualitativas. A partir de este, de forma aleatoria, se coordinaron citas con empresas y bajo el criterio del principio de saturación se concluyó con la realización de entrevistas una vez que la información obtenida de la muestra no presentó novedades en los datos y, por ende, se cumplió con el objetivo general (Guest et al., 2006). Las compañías seleccionadas pertenecen a distintas industrias, tal como se detalla en la tabla 1 . 
Así mismo, se consideraron empresas con distintos niveles de desempeño exportador, en las que el 6,25 $\%$ de los participantes posee un nivel poco exitoso, mientras que el $56,25 \%$ es medianamente exitoso y, finalmente, el 37,5 \% restante posee un nivel altamente exitoso. La clasificación de éxito la brinda en la base de datos otorgada por Procomer.

Tabla 1. Porcentaje de participación en la muestra según el tipo de industria

\begin{tabular}{|c|c|}
\hline Tipo de industria & Porcentaje de participación \\
\hline Agrícola & $12,50 \%$ \\
\hline Muebles y aparatos de alumbrado & $12,50 \%$ \\
\hline Productos minerales no metálicos & $6,25 \%$ \\
\hline Eléctrica y electrónica & $6,25 \%$ \\
\hline Plástico & $6,25 \%$ \\
\hline Química & $31,25 \%$ \\
\hline Alimentaria & $6,25 \%$ \\
\hline Equipo de precisión y médico & $6,25 \%$ \\
\hline Maderera & $6,25 \%$ \\
\hline Textiles, cuero y calzado & $6,25 \%$ \\
\hline
\end{tabular}

Fuente. Elaboración propia.

Para el procesamiento y el análisis de la información recolectada fue necesario transcribir las entrevistas realizadas que se grabaron en audio, luego se construyó una lista de códigos dentro de los cuales se encuentran actividades de mercadeo digital y tradicional. El primero incluye redes sociales, email marketing y página web. Además, se estableció el nivel de beneficio obtenido de estas actividades para la empresa y los diferentes actores relacionados con esta, así como formas para medir los resultados, la finalidad del uso de mercadeo digital y los tipos de redes sociales utilizados. Todos estos códigos luego sirvieron para el procesamiento de la información con el software AtlasTI8 al realizar un análisis por medio de la relación de las variables.

\section{Resultados}

T as compañías participantes del estudio fueron Lcreadas, en promedio, hace 57 años, y poseen una media de 349 colaboradores. Los entrevistados son profesionales que, en promedio, tienen diez años de laborar en la organización, específicamente en el departamento de mercadeo o comercial, el cual, en promedio, se encuentra conformado por siete colaboradores.
Como parte de la entrevista realizada se les consultó a los participantes cuáles actividades de mercadeo desarrollan en la organización. Las respuestas a este interrogante indican que las compañías utilizan medios tanto tradicionales como digitales, tal como se muestra en la figura 1 ; en esta se muestra una nube de palabras con las principales respuestas. Además, en la tabla 2 se menciona la cantidad de veces que fue mencionada cada actividad. 
Tabla 2. Principales actividades de mercadeo que se realizan en las organizaciones según la cantidad de veces que fueron mencionadas

\begin{tabular}{|c|c|}
\hline Actividad de mercadeo & $\begin{array}{c}\text { Cantidad de veces que se } \\
\text { mencionó }\end{array}$ \\
\hline Eventos & 5 \\
\hline Ventas & 5 \\
\hline Televisión & 4 \\
\hline Redes sociales & 4 \\
\hline Mercadeo & 4 \\
\hline Puntos de venta & 4 \\
\hline Estrategias & 3 \\
\hline Publicidad & 3 \\
\hline Material POP & 3 \\
\hline Ferias & 3 \\
\hline Radio & 3 \\
\hline
\end{tabular}

Fuente. Elaboración propia.

Figura 1. Principales actividades de mercadeo que se realizan en las organizaciones

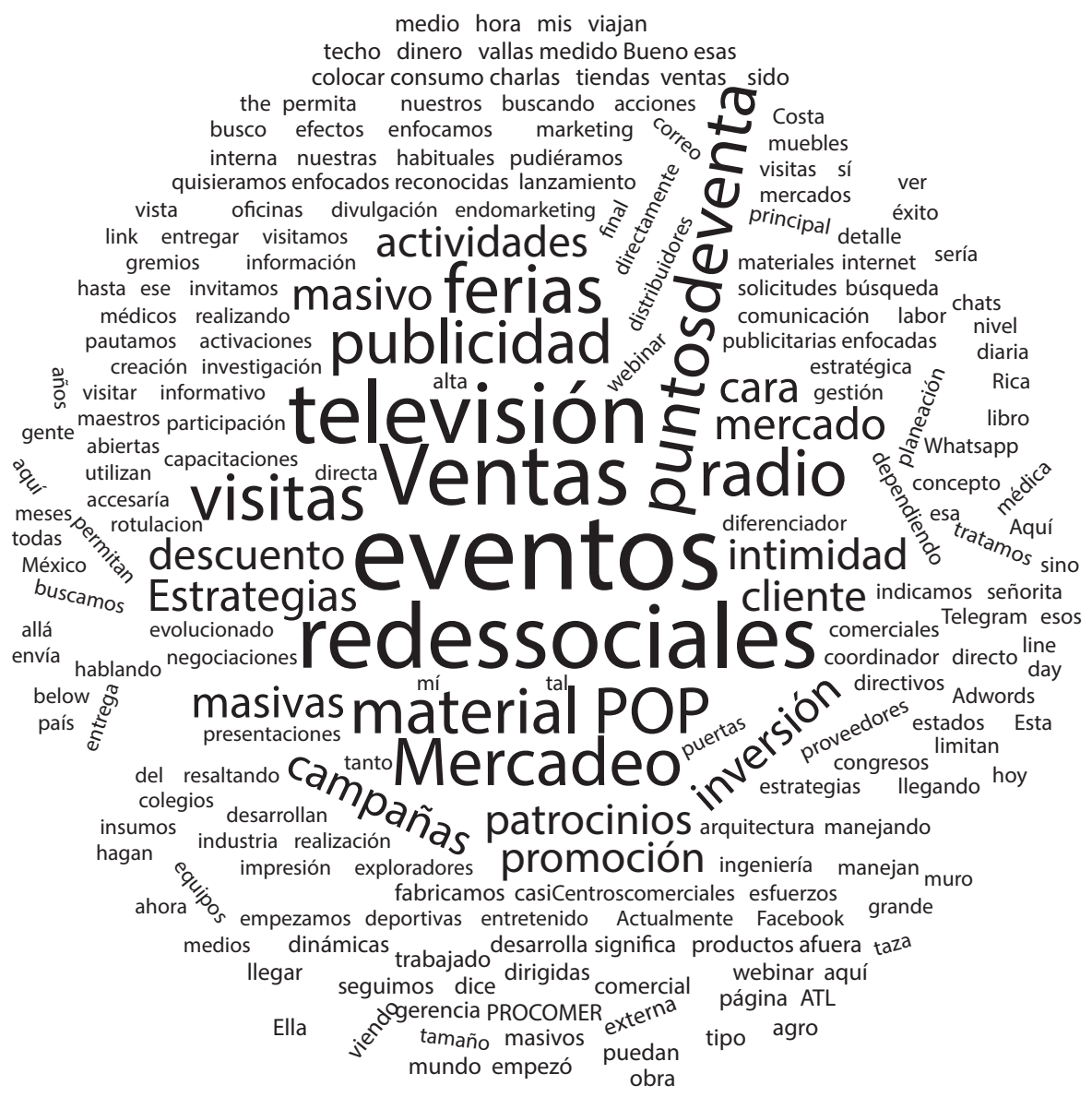

Fuente. Elaboración propia. 
De acuerdo con la cantidad de citas obtenidas, entre los principales beneficios obtenidos de estas actividades se encuentra el incremento en la cantidad de clientes _ocho-, la promoción de la marca - ocho-, el desarrollo de sus productos -ocho-, el conocimiento de los clientes cinco-, la recordación de la empresa — seis- y el posicionamiento, la credibilidad y la estabilidad seis-. Por su parte, los entrevistados comentaron que la medición de estos beneficios se mensura, principalmente, a través de la cantidad de clientes - ocho- o de la cantidad de personas - once-, reconocimiento de los productos _ocho-, ventas —ocho-, crecimiento — tres- y retorno de la inversión - dos-.
En lo referente a las herramientas de mercadeo digital, estas se utilizan desde hace cinco años, en promedio. De los dieciséis entrevistados que participaron en el estudio, todos afirmaron utilizar, al menos, una herramienta de este tipo. Además, doce de ellos mencionaron que estas las utilizan funcionarios de la organización a nivel interno, sin la necesidad de subcontratar una entidad externa. En la tabla 3 se detallan las principales herramientas de mercadeo digital que utilizan las organizaciones participantes, en la que se señala la cantidad de veces que se mencionó cada una de ellas; además se muestra una cita representativa.

Tabla 3. Principales herramientas de mercadeo digital utilizadas en las organizaciones según la cantidad de veces que fueron mencionadas

\begin{tabular}{|c|c|l|}
\hline Herramienta & Cantidad de citas & \multicolumn{1}{c|}{ Cita preventiva } \\
\hline Pagina web & 22 & $\begin{array}{l}\text { «La pagina web (...) es una forma de promoción de productos que } \\
\text { también se ha establecido como una nueva forma de hacer negocios (...) } \\
\text { tiene un enlace para contacto, entonces esa información me llega a mi } \\
\text { directamente» }\end{array}$ \\
\hline Redes sociales & 12 & $\begin{array}{l}\text { «Las redes sociales han venido a ser un apoyo muy grande (...) tenemos } \\
\text { a la vieja escuela, verdad, que son nuestros clientes de más de ciencuenta } \\
\text { años y que alguno que pues por un tema ahí de modas se han ido teniendo } \\
\text { que ir actualizando (..) viene una nueva generación que ocupamos que } \\
\text { también sean nuestros futuros clientes, ¿verdad? Actuales y futuros } \\
\text { clientes, que se mantengan con nosotros a través del tiempo y con eso sí } \\
(\ldots) \text { ha venido a ser muy muy importante» }\end{array}$ \\
\hline Email marketing & 2 & $\begin{array}{l}\text { «Nosotros trabajamos mucho por correo, se mandan ofertas o se hace } \\
\text { investigación de mercados, donde trambién tratamos de contactar } \\
\text { bastantes clientes por este medio.» }\end{array}$ \\
\hline
\end{tabular}

Fuente. Elaboración propia.

Con respecto a las redes sociales, un $81,25 \%$ de los participantes mencionó que utiliza Facebook, mientras que ocho afirmaron que también emplean Instagram, seguida de Youtube, Google y Linkedin. Además, se obtuvo un total de veintidós citas relacionadas con la página web de la empresa, en donde trece de los dieciséis entrevistados afirmaron que la utilizan. En lo que respecta a la medición de los resultados obtenidos a partir de estas herramientas, de los dieciséis entrevistados, cinco personas mencionaron que utilizan los reportes que brinda
Facebook sobre el alcance de las publicaciones realizadas, mientras que tres mencionaron que no miden el desempeño de las herramientas de mercadeo digital. Por su parte, se identificaron ocho citas en las que los entrevistados afirman que utilizan otras plataformas o instrumentos para medir los resultados obtenidos, tales como Google Analytics — dos-, Balance Scorecard -uno-, Adobe Analytics uno-, Power BI —uno-, Manychat —uno- y Mailchimp — dos-. 
En cuanto a los fines para los que utilizan estas herramientas, cuatro de los dieciséis participantes mencionaron que el objetivo principal es promocionar los productos de la empresa, tal como lo comentó uno de ellos: «Para promocionar, lo que sería promocionar productos, nos gusta hacer técnicas como para llamar a los clientes a participar. Hacemos mucho concurso y lanzamientos de producto, más que todo como para dar a conocerlos a la gente» (comunicación personal). Por otra parte, tres de los dieciséis entrevistados mencionaron que utilizan estas herramientas para mantener la comunicación con sus clientes y tratar de conquistar a un público más joven, tal como se detalla a continuación:

Un poquito es para tratar de llegarle a la gente joven, porque muchos de los jóvenes ya no ven televisión, pero hemos tratado de buscarle la alternativa de cómo llegarles. Principalmente, lo que tratamos de hacer es comunicación, que la gente tenga acceso a la marca independientemente de la edad que tiene. (Comunicación personal)
Así mismo, tres de las dieciséis personas indicaron que también utilizan estas herramientas para llevar a cabo el proceso de ventas, lo que incluye servicio preventa y posventa.

De igual manera, se les consultó a los participantes el nivel de beneficio que han obtenido a partir del mercadeo digital en distintos aspectos de la organización (véase la Tabla 4). Los datos indican que quince de dieciséis entrevistados afirman que las herramientas de mercadeo digital les han sido beneficiosas y muy beneficiosas para realizar actividades relacionadas con la competencia, al igual que para la búsqueda de nuevos clientes. En contraparte, existen algunas áreas en las que estas herramientas les han otorgado poco beneficio, tales como la gestión de canales de distribución y proveedores.

Tabla 4. Nivel de beneficio obtenido en diferentes aspectos a partir del mercadeo digital según la cantidad de citas obtenidas

\begin{tabular}{|c|c|c|c|c|}
\hline \multirow[b]{2}{*}{ Aspecto } & \multicolumn{3}{|c|}{ Nivel de beneficio } & \multirow[b]{2}{*}{ Cita preventiva } \\
\hline & $\begin{array}{c}\text { Poco } \\
\text { beneficioso }\end{array}$ & Beneficioso & $\begin{array}{c}\text { Muy } \\
\text { beneficioso }\end{array}$ & \\
\hline $\begin{array}{c}\text { Aliados } \\
\text { estrategicos }\end{array}$ & 8 & 3 & 5 & $\begin{array}{l}\text { «emos hecho muchos proyectos (...) no se que tanto ha } \\
\text { resultado por el marketing digital (...) no le daría tanto el } \\
\text { mérito al marketing digital sino que tal vez han sido otros } \\
\text { intereses, pero por supuesto lo que eso ha ayudado también.» }\end{array}$ \\
\hline $\begin{array}{l}\text { Canales de } \\
\text { distribución }\end{array}$ & 10 & 2 & 4 & $\begin{array}{l}\ll \text { No abarca mucho, por que nosotros no manejamos como } \\
\text { pedidos online, por decirte algo . Entonces no, no nos abarca } \\
\text { mucho.» }\end{array}$ \\
\hline $\begin{array}{l}\text { Clientes actuales } \\
\text { y su relación con } \\
\text { la empresa }\end{array}$ & 3 & 5 & 8 & $\begin{array}{l}\text { «Porque una vez mas la gente está a la distancia de un click, } \\
\text { se siente mas cercano, se siente parte de la compañia, siente } \\
\text { que esta informado continuamente. No tiene que esperar un } \\
\text { anuncio de televisión para darse cuenta de una promoción o } \\
\text { del nuevo producto que se esta lanzando (...) se van dando } \\
\text { cuenta de todas las tendencias.» }\end{array}$ \\
\hline Competencia & 1 & 6 & 9 & $\begin{array}{l}\ll \text { Nosotros tratamos siempre de estar muy al pendiente de } \\
\text { ellos, por decirte algo, para ver que sacan y nosotros tratar } \\
\text { de ofrecer lo mismo para abarcar todo el mercado que esta a } \\
\text { nuestro alcance. Entonces, por ejemplo, los medios digitales } \\
\text { nos han permitido estar como mas atentos a lo que ellos hacen } \\
\text { y nosotros tirar una propuesta para eso, di, no quedamos fuera } \\
\text { del nicho de mercado.» }\end{array}$ \\
\hline $\begin{array}{l}\text { Diversificación de } \\
\text { productos }\end{array}$ & 3 & 6 & 7 & $\begin{array}{l}\text { «Nos permite ver que es lo que está en tendencia, que es lo } \\
\text { que está en el mercado y que es lo que los clientes quieren.» }\end{array}$ \\
\hline
\end{tabular}




\begin{tabular}{|c|c|c|c|l|}
\hline $\begin{array}{c}\text { Estrategias de } \\
\text { mercado para } \\
\text { ventas locales } \\
\text { internacionales }\end{array}$ & 4 & 5 & 7 & $\begin{array}{l}\text { «Permite darle un mayor seguimiento a las cosas y en caso } \\
\text { de que algo no este funcionando (...) cambiar la publicación } \\
\text { o detenerla. Ya cuando estamos hablando (...) de medios } \\
\text { tradicionales, ya por ejemplo cuando el periódico salió, ya salió, } \\
\text { entonces no se pudo hacer nada. Entonces da la posibilidad de } \\
\text { ser rectivos y proactivos.» }\end{array}$ \\
\hline Nuevos clientes & 1 & 7 & 8 & $\begin{array}{l}\text { «Yo creo que es beneficioso para los muchachos jóvenes, } \\
\text { para poder accesarlos y que tengan conocimiento de la } \\
\text { marca. Nosotros vamos siempre detrás de un porcentaje de } \\
\text { mercado.» }\end{array}$ \\
\hline Nuevos negocios & 5 & 3 & 8 & $\begin{array}{l}\text { «Nos han dado oportunidad de participar inclusive en ferias } \\
\text { internacionales (...) por que lo vieron en redes sociales (...) } \\
\text { por que lo vieron y se interesaron, y nos buscan.» }\end{array}$ \\
\hline Proveedores & 11 & 2 & 3 & $\begin{array}{l}\text { «No se enteran mucho de nosotros (...) creo que nos han visto } \\
\text { más por los medios tradicionales.» }\end{array}$ \\
\hline
\end{tabular}

Fuente. Elaboración propia.

Con relación al desempeño exportador, de los dieciséis entrevistados nueve participantes mencionaron que entre los principales destinos de exportación se encuentran los países de Centroamérica. Además, diez personas comentaron que, inicialmente, sus organizaciones no nacieron con la idea de exportar, sino que esto fue una decisión tomada a raíz de diferentes motivos, tal como se menciona en la siguiente cita:

Yo creo que, hace cincuenta años, tal vez la visión no era tan amplia [...] A raíz del paso de los años, se empezaron a ver las oportunidades y fuimos una empresa que notó esas oportunidades, y nos sirvió, y hoy se ha convertido en una empresa que está en toda la región. (Comunicación personal)

Al analizar el desempeño exportador, según los resultados obtenidos, nueve de los dieciséis entrevistados mencionaron que la calidad de los productos es uno de los principales factores que justifican el nivel de exportación. Además, se identificaron algunas citas relacionadas con otros aspectos, tales como las características del producto ofertado - seis - y la forma en que lo distribuyen — cinco—, además de la investigación —dos—y el precio - dos-.
Con respecto a las actividades de mercadeo que realizan las empresas para potenciar su desempeño exportador, seis de las dieciséis personas mencionaron que las visitas a los clientes actuales o potenciales son fundamentales para el logro de los objetivos, tal como lo mencionó una de ellas: «Para nosotros es fundamental ver al cliente, cara a cara, al dueño de la empresa» (comunicación personal). Otro de los entrevistados mencionó que esto es con el fin de «ver la estructura de él, ver qué es lo que requiere el cliente, sus necesidades, y si es factible que uno le venda» (comunicación personal).

Sumado a lo anterior, se identificaron seis citas en las que los participantes mencionaron que las diferencias culturales, legales y políticas constituyen barreras que obstaculizan estos esfuerzos; uno de ellos indicó: «Todos los países son diferentes, con diferentes culturas $[. .$.$] nuestro servicio debe ser el mismo en$ los países en los que estamos [...] si encontramos retos a nivel de estrategia, a nivel de aceptación». No obstante, también se identificaron cinco citas en las que se indica que las compañías no han tenido ningún tipo de barrera para desarrollar estas actividades. 
En el mismo orden de ideas, de los dieciséis entrevistados trece indicaron que los esfuerzos de mercadeo que se realizan en las organizaciones varían según el país al que se desee exportar, tal como lo mencionó el siguiente entrevistado cuando se le realizó la consulta: «Yo creo que depende mucho del país, porque todos los mercados cambian mucho. El desarrollo digital de muchos mercados no es el mismo, hay mercados todavía muy tradicionales. Entonces sí, hay que ajustarse a los mercados sin duda alguna» (comunicación personal).
Finalmente, doce participantes afirmaron que, efectivamente, sí existe una relación entre las herramientas de mercadeo que utilizan y el desempeño exportador de la empresa, en la que uno de los participantes respondió el interrogante de la siguiente forma:

Por supuesto, no es lo mismo activar a no hacer nada... Parte de los buenos resultados que hemos tenido en los últimos años es porque antes no se hacía nada y ahora estamos haciendo muchas cosas, y eso nos ha encadenado todos los resultados positivos que tenemos hoy. Entonces, definitivamente sí.

\section{Discusión y conclusiones}

¿s resultados obtenidos permiten concluir existe una posible relación entre las herramientas de mercadeo tradicional y digital utilizadas por grandes empresas y su desempeño exportador, tomando en consideración que este es un país en vía de desarrollo. Con respecto a las actividades de mercadeo que se desarrollan en las organizaciones para potenciar su internacionalización, se concluye que existe una predominancia en el uso de herramientas tradicionales, entre las cuales destacan la organización de eventos, actividades en puntos de venta y ferias, televisión, radio y materiales POP — por sus siglas en inglés, point of purchase-, tal como se mostró en la tabla 1. En el caso de los eventos, los entrevistados destacan la importancia de entablar un contacto presencial con los clientes, de manera que aumente la confianza a la hora de realizar los negocios de exportación.

Sin embargo, los resultados señalan que el mercadeo digital también se utiliza en conjunto con el tradicional, lo cual respalda lo mencionado por García-Marín (2015), quien afirma que las compañías deben utilizar medios tanto tradicionales como digitales, debido a que no todos los consumidores están familiarizados con el manejo de estas tecnologías. Sin embargo, también es importante tomar en cuenta el mercado meta de la organización, con el fin de desarrollar esfuerzos de mercadeo que se adapten a sus características. Agregado a lo anterior, cabe destacar que todas las empresas participantes utilizan, al menos, una herramienta de mercadeo digital, lo cual apoya las afirmaciones de Yogesh et al. (2019), quienes consideran que el desarrollo de nuevas tecnologías y las herramientas de comunicación digital incitan a las organizaciones a transformar la manera en que comercializan sus productos. De igual forma, a pesar de que existen múltiples herramientas de mercadeo digital, las organizaciones prefieren utilizar la página web, las redes sociales - principalmente Facebook e Instagram-y el email marketing.

Agregado a lo anterior, se concluyó que cada vez son más las compañías que manejan las herramientas de mercadeo digital a nivel interno dentrola organización, debido a la importancia de estas para el logro de los objetivos. Con el paso del tiempo, las compañías han apostado a capacitar a sus propios colaboradores en el uso de estas herramientas y dejan de lado servicios de outsourcing, en específico las agencias publicitarias. No obstante, es importante destacar que existe un desconocimiento de los distintos instrumentos o plataformas que se utilizan para medir los resultados 
obtenidos a partir de estas herramientas, lo cual produce que se dificulte la cuantificación de los esfuerzos realizados.

En el mismo orden de ideas, se determinó que el principal fin para el que se utilizan las herramientas de mercadeo digital es la promoción de los productos, dada la importancia de estas en la cotidianeidad actual de los seres humanos, quienes en su mayoría tienen acceso a este tipo de plataformas y las utilizan a diario. Además, los resultados permiten concluir que estas herramientas son de gran utilidad para que las organizaciones puedan abarcar nuevos segmentos de mercado, principalmente en temas generacionales para empresas que desean atraer un mercado más joven, el cual se encuentra inmerso en el uso de estas redes.

De igual manera, otro de los beneficios es que permite mantener una relación más cercana con sus clientes y conocer mejor sus necesidades y deseos, en un menor tiempo. Lo anterior confirma lo expuesto por Gómez (2013), quien sostiene que este tipo de herramientas permiten que las empresas se comuniquen con sus clientes actuales $\mathrm{y} / \mathrm{o}$ potenciales de una mejor forma.

Con respecto a la competencia, los resultados obtenidos indican que las herramientas de mercadeo digital han sido muybeneficiosas, puesto que permiten conocer qué realizan los principales competidores y hacia dónde se dirigen sus esfuerzos, de forma que en la organización se puedan tomar decisiones con miras a obtener una ventaja competitiva. De igual modo, el nivel de beneficio es el mismo para la diversificación de los productos, puesto que gracias a estas herramientas las organizaciones han logrado conocer las necesidades y los deseos de sus clientes de una manera más cercana, información clave en el desarrollo de bienes y servicios que satisfagan dichas necesidades y deseos.

Así mismo, estas herramientas han sido muy beneficiosas para la búsqueda de nuevos clientes, tal como lo indica Anato (2006), quien expresa que las organizaciones deben esforzarse por utilizar los medios digitales con el fin de desarrollar una comunicación de tipo personal y, de esta forma, establecer relaciones a largo plazo con los clientes mediante la interacción en comunidades virtuales. No obstante, esto hace mayor énfasis en los clientes individuales, es decir, los consumidores finales, puesto que los clientes propios de exportación prefieren medios más tradicionales, tal como se explicó. Agregado a lo anterior, este tipo de herramientas han sido muy beneficiosas en la identificación y el desarrollo de nuevos negocios para las compañías, lo cual se traduce en una mayor rentabilidad para las organizaciones.

Por otra parte, existen algunos aspectos en los que estas plataformas no han sido provechosas, como, por ejemplo, en la comunicación que mantienen las organizaciones con los proveedores actuales o potenciales y la gestión que maneja la compañía con respecto a los canales de distribución, en el que se ha obtenido poco beneficio. Por otra parte, en la búsqueda y el establecimiento de alianzas estratégicas se obtuvo cierto beneficio producto de estas plataformas, puesto que permiten identificar organizaciones potenciales que persigan un objetivo afín.

Con respecto al desempeño exportador, se evidenció que, actualmente, las compañías buscan exportar con el propósito de aumentar sus posibilidades de éxito; lo anterior mediante la identificación $y$ el involucramiento en nuevos mercados. Esta conclusión apoya lo expuesto por Kotler (2002), quien explica que, por lo general, las empresas buscan incrementar su nivel de ventas mediante la búsqueda de nuevos negocios. Cabe destacar que la mayoría de estas organizaciones no fueron fundadas con la idea de exportar, sin embargo, lo han tenido que realizar con el fin de aprovechar un mercado potencial más allá de sus fronteras en las que, generalmente, las oportunidades no son tan amplias.

De igual forma, cabe destacar que uno de los principales factores que ha sido clave para esto es la calidad de los productos ofertados por estas compañías localizadas en territorio costarricense, seguido del producto en sí y la forma en la que se distribuye. 
Ahora bien, en cuanto a las actividades de mercadeo realizadas para la promoción de las exportaciones, se concluyó que las visitas a los clientes juegan un papel protagónico en el proceso requerido para el cierre de la venta, en la que los negocios se dialoguen cara a cara y se genere una mayor confianza e interacción con el cliente. Finalmente, los resultados sugieren que existen ciertas barreras para desarrollar estas actividades, principalmente en cuanto al idioma y las demás características culturales de cada país, sumadas a la situación política y económica.

Por tanto, la mayoría de entrevistados comentó que es necesario adaptarlas a las características propias de cada país, tal como lo afirman Galindo y Viridiana (2015), quienes comentan que el hecho de ampliar más allá de las fronteras el segmento al que la empresa dirige sus operaciones es un reto que las compañías deben superar con una estrategia adecuada, la cual debe considerar aspectos culturales, políticos, económicos y sociales. Además, es necesario investigar el contexto específico en el que se desarrolla la organización, en aras de que la estrategia se adapte en mayor medida a la empresa.

En contraparte, la fracción de participantes que afirmó no haber tenido que lidiar con estas barreras, en su mayoría cuenta con representantes de la compañía o aliados estratégicos en los países a los que exportan o desean hacerlo, lo cual agiliza la negociación, puesto que se conocen estos aspectos propios de cada país y de cada organización, minimizando los esfuerzos que se deben realizar en comparación a los negocios que se establecen desde cero.

Dado lo anterior, se logra identificar que en las empresas estudiadas se ha logrado un equilibrio entre mantener las actividades de mercadeo tradicional e incursionar en el uso del mercado digital. Así, se logra demostrar que ambos son beneficiosos para conseguir un mejor rendimiento en su nivel de desempeño exportador, tomando en consideración el sector en el que se encuentren y el tipo de clientes a los que se dirigen.

\section{Referencias}

Agüero, L. (2014). Estrategia de fidelización de los clientes (Trabajo de grado). Universidad de Cantabria. Cantabria, España. Recuperado de http://bit.ly/2BwqSkv

Anato, M. (2006). El uso de los instrumentos clásicos del marketing y la tecnología digital en turismo. Estudios y Perspectivas en Turismo, 15(1), 19-40.

Argüelles-Vélez, M.; Benavides-González, C. (2008). Conocimiento y crecimiento económico: una estrategia para los países en vías de desarrollo. Revista de Economía Mundial, (18), 65-77.

Asare, T.; Brashear, J.; Jun, K. (2013). The relationship between supplier development and firm performance: the mediating role of marketing process improvement. Journal of Business \& Industrial Marketing, 28(6), 523-532. DOI: https://doi. org/10.1108/JBIM-04-2013-0100

Bala, C.; Gomathi, J. (2017). Digital marketing and its challenges. Airo International Research Journal, 12, 2-17.

Bertaux, D. (1981). Biography and society: the life history approach in the social. Londres: Sage.

Bianchi, C.; Mathews, S. (2016). Internet marketing and export market growth in Chile. Journal of Business Research, 69(2), 426434. DOI: https://doi.org/10.1016/j.jbusres.2015.06.048

Castellano, S.; Urdaneta, A. (2015). Estrategias de mercadeo verde utilizadas por empresas a nivel mundial. TELOS. Revista de Estudios Interdisciplinarios en Ciencias Sociales, 17(3), 476494.

Cobo, F.; González, L. (2007). Las implicaciones estratégicas del marketing relacional: fidelización y mercados ampliados. Anuario Jurídico y Económico Escurialense, (40), 543-568.

Cortés, S. (2011). Marketing digital como herramienta de negocios para pymes (Seminario para optar al título de Ingeniero Comercial). Universidad de Chile. Santiago, Chile. Recuperado de http://bit.ly/2N3ENUz

Dijk, M. van. (2002). The determinants of export performance in developing countries: the case of indonesian manufacturing. Eindhoven Centre for Innovation Studies, Eindhoven, Países Bajos. Recuperado de http://bit.ly/2UwwDtd

Diez, F.; Blanco, A.; Prado, C. (2019). Research challenges in digital marketing: sustainability. Sustainability, 10(11), 2839 DOI: https://doi.org/10.3390/su11102839

Galindo, M.; Viridiana, R. (2015). México ¿cómo vamos? Exportaciones. en Serie de en Estudios Económicos, Vol. 1. Mexico DF: México ¿cómo vamos? 
García-Marín, I. (2015). La era del mercadeo digital. Debates IESA, XX(1), 48. Recuperado de http://bit.ly/2JhAh3I

García, F.; Avella, L. (2007). Intensidad exportadora y percepción de barreras a la exportación: un estudio de casos. Investigaciones Europeas de Dirección y Economía de la Empresa, 13(3), 93-106.

Gervase, C. (2009). What is marketing? CustomerThink. Recuperado de https://bit.ly/3eKgYAs

Gómez, J. (2013). El marketing digital y las estrategias on-line de las microempresas colombianas (Tesis de grado). Universidad Militar Nueva Granada. Bogotá, Colombia. Recuperado de http://bit.ly/2JfQgix

Granada, D. (2012). Costumer relationship management en las organizaciones (Tesis de grado). Universidad Militar Nueva Granada. Bogotá, Colombia. Recuperado de http://bit. ly/33Ry8DA

Grávalos, D. (2013). La calidad de una página web como herramienta de comunicación. Estudios sobre el Mensaje Periodístico, 19, 253-261. DOI: https://doi.org/10.5209/rev_ESMP.2013. v19.42032

Gregory, G.; Viet-Ngo, L.; Karavdic, M. (2019). Developing e-commerce marketing capabilities and efficiencies for enhanced performance in business-to-business export ventures. Industrial Marketing Management, 78, 146-157. DOI: https://doi.org/10.1016/j.indmarman.2017.03.002

Guest, G.; Bunce, A.; Johnson, L. (2006). How many interviews are enough? An experiment with data saturation and variability. Field Methods, 18(1), 59-82. DOI: https://doi. org/10.1177\%2F1525822X05279903

Hernández, R.; Fernández, C.; Baptista, M. (2014). Metodología de la investigación. (6a ed.). México: McGraw-Hill Education. Recuperado de http://bit.ly/2pFWth0

Hurtado, A.; Castillo, M. (2013). Influencia de las barreras a la exportación sobre el compromiso exportador y su incidencia en los resultados internacionales. Revista Escuela de Administración de Negocios, (75), 38-55. DOI: https://doi. org/10.21158/01208160.n75.2013.770

Jiménez, A. (2010). Los procesos de globalización e integración económica: retos y oportunidades para el mercadeo internacional de las empresas. Revista Venezolana de Análisis de Coyuntura, 16(2), 95-113.

Khan, G.; Maryum-Umar, A.; Zaman, N.; Ahmad, E.; Shoukat, Y. (2012). Export, import and economic growth nexus: Time series evidence from Pakistan. World Applied Sciences Journal, 18(4), 538-542.

Kotler, P. (2002). Dirección de marketing: conceptos esenciales. México: Pearson Educación.

Ledermana, D.; Olarreaga, M.; Zavala, L. (2016). Export promotion and firm entry and survival in export markets. Canadian Journal of Development Studies, 37(2), 142-158. DOI: https://doi.org/10.1080/02255189.2016.1131671
Maqueira, J. M.; Bruque, S. (2012). Marketing 2.0: el nuevo marketing en la web de las redes sociales. México: Alfaomega.

Mohasoa, K. (2014). Marketing theory: experiential vs. traditional marketing. University of the Witwatersrand, Johannesburgo, Sudáfrica. Recuperado de http://bit.ly/2JgiLg3

Moreno, O. (2007). El marketing internacional como herramienta indispensable para una exportación efectiva. Ciencia en su PC, (2), 1-10.

Moschini, S. (2012). Claves del marketing digital. La nueva comunicación empresarial en el mundo 3.0. Barcelona: La Vanguardia Ediciones. Recuperado de http://bit.ly/35XyDOs

Peláez, M. J.; Rodenes, M. (2009). La internacionalización de empresas: relación entre el capital social, las tecnologías de la información relacional y la innovación. Semestre Económico, $12(25), 111-138$.

Procomer (Promotora de Comercio Exterior). (2019). Estadísticas de comercio exterior Costa Rica 2016. https://bit. ly/38JsYP2

Puente-Riofrío, M.; Andrade-Domínguez, F. (2016). Relación entre la diversificación de productos y la rentabilidad empresarial.RevistaCienciaUnemI,9(18),73-80.DOI:https:// doi.org/10.29076/issn.2528-7737vol9iss18.2016pp73-80p

Raluca, D. (2016). Blending traditional and digital marketing. Bulletin of the Transilvania University of Brasov, 9(58), 51-56.

Rodríguez, C.; Camargo, P.; González, J.; Gorrin, J. (2015). Ventajas y desventajas del marketing digital. Revista convicciones, 2(3), 24-30.

Tan, Q.; Sousa, C. (2015). Leveraging marketing capabilities into competitive advantage and export performance. International Marketing Review, 32(1), 78-102. DOI: https://doi. org/10.1108/IMR-12-2013-0279

Vanegas, A. (2012). Introducción al e-marketing para profesionales de mercadeo (Tesis de grado). Institución Universitaria Esumer. Medellín, Colombia. Recuperado de http://bit.ly/31CkanI

Vásquez, G. (2009). Los canales de distribución y el valor para el consumidor. Temas de Management. Recuperado de http:// bit.ly/2ByUhdL

Yamin, A. (2017). Impact of digital marketing as a tool of marketing communication: a behavioral perspective on consumers of Bangladesh. American Journal of Trade and Policy, 4(3), 117-122. DOI: https://doi.org/10.18034/ajtp. v4i3.426

Yogesh, S.; Sharaha, N.; Roopan, S. (2019). Digital marketing and its analysis. International Journal of Emerging Technology and Innovative Engineering, 5(7), 469-474 